\title{
Epidemiological evaluation of an Acinetobacter baumannii outbreak observed at an intensive care unit
}

\author{
Tuğba K. Atik, MD, Bülent Atik, MD, Osman Kilinç, MD, Bayhan Bektöre, MD, Hülya Duran, MD, Burak M. Selek, MD, \\ Nihan Ceken, MD, Orhan Baylan, MD, Mustafa Özyurt, MD.
}

\begin{abstract}

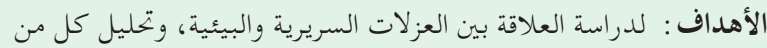

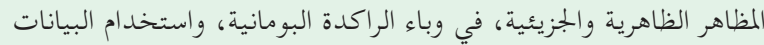

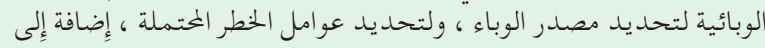

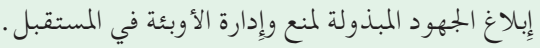

الطريقة : تم عزل الراكدة البومانية من 5 عينات سريرية في مستشفى سلطان

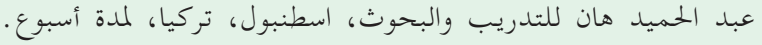

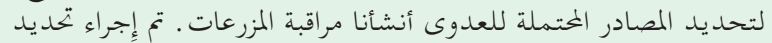

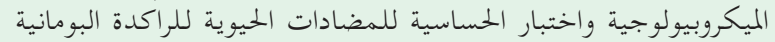

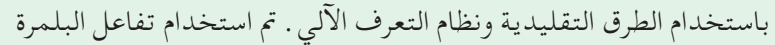

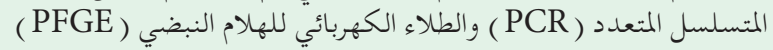

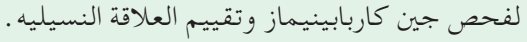

النتائج: من بين العينات البيئية، لوحظ نمو البكتيريا في 3 من ثقافات

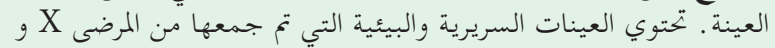

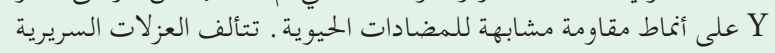

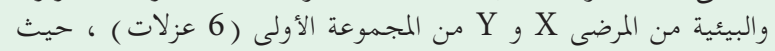

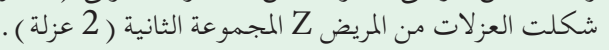

الخاتمة: لقد اكتشفنا أن جميع العزلات المرتبطة بالفيروس تمتوي على

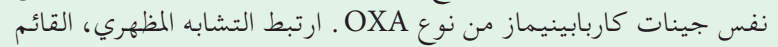

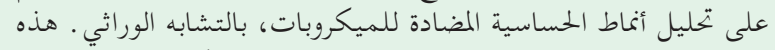

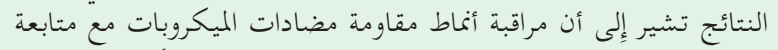

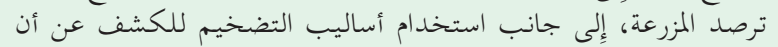

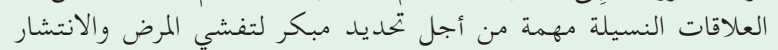

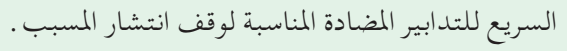

Objectives: To reveal the relationship between clinical and environmental isolates, analyzing both phenotypic and molecular aspects, in an Acinetobacter baumannii (A. baumannii) epidemic, and to use the epidemiological data to determine the source of the epidemic, to identify potential risk factors, and inform the effort to prevent and manage future epidemics.

Methods: Acinetobacter baumannii was isolated from 5 clinical samples in Sultan Abdulhamid Han Training and Research hospital, Istanbul, Turkey, for a week period. To determine potential sources of infection we established cultures surveillance. Microbiological identification and antibiotic susceptibility testing of $A$. baumannii were performed using conventional methods and automated identification system. Multiplex polymerase chain reaction (PCR) and pulsed-field gel electrophoresis (PFGE) were used for carbapenemase gene screening and clonal relationship evaluation.

Results: Among the environmental samples, bacterial growth was observed in 3 of the sample cultures. Clinical and environmental samples collected from patients $\mathrm{X}$ and $Y$ had phenotypically similar antibiotic susceptibility patterns. The clinical and environmental isolates from patients $\mathrm{X}$ and $\mathrm{Y}$ comprised the first cluster (6 isolates), the isolates from patient $\mathrm{Z}$ formed the second cluster (2 isolates).

Conclusion: We detected that all outbreak-related isolates contained the same OXA-type carbapenemase genes. Phenotypic similarity, based on the analysis of antimicrobial susceptibility patterns, was correlated with genotypic similarity. These results suggest that monitoring antimicrobial resistance patterns with daily culture surveillance follow-ups, coupled with the use of amplification based methods to detect that clonal relationships are important for the early identification of outbreaks and rapid deployment of proper countermeasures to halt the spread of the causative agent.

Saudi Med J 2018; Vol. 39 (8): 767-772

doi: 10.15537/smj.2018.8.22431

From the Department of Microbiology (K. Atik, Kilinç), and from the Department of Anesthesiology and Reanimation clinic (Atik), Balikesir Atatürk City hospital, from the Department of Microbiology (Duran, Cleken), Balikesir Public hospital, Balikesir, from the Department of Microbiology (Bektöre), Kars Harakani Public hospital, Kars, and from the Department of Microbiology (selek, Baylan, Özyurt), Sultan Abdulhamid Han Training and Research hospital, Health Sciences University, İstanbul, Turkey.

Received 19th March 2018. Accepted 22nd July 2018.

Address correspondence and reprint request to: Dr. Tü̆ba K. Atik, Department of Microbiology, Balıkesir Atatürk City hospital, Balıkesir, Turkey. E-mail: tkulaatik@gmail.com

ORCID ID: orcid.org/0000-0002-2433-1977 
A substantial increase in disease outbreaks caused by non-fermenter bacilli such as Acinetobacter baumannii (A. baumannii) has been observed in intensive care units (ICUs). ${ }^{1,2}$ The ability of $A$. baumannii to remain viable for long periods and its rapid dissemination through cross-contamination in hospital environments are both important considerations when studying the association of $A$. baumannii with health care-associated infections (HAI) and epidemics. ${ }^{1-3}$

In hospitals, respiratory equipments and devices are the most common surfaces for the colonization of Acinetobacter species; others include the bed rails, bedside table, bed linen, infusion tubing, bedpans, urinals, monitors, knobs, buttons and other medical equipments and "high frequency" touch surfaces. ${ }^{1-4}$ Due to high rates of transfer by contact with skin, both patients and the hands of health care workers are important infection sources when infection control precautions are ignored during epidemics. 3

Beyond its role as an epidemic causal factor, A. baumannii is also clinically important due to its ability to rapidly transfer antimicrobial resistance genes and produce resistant isolates. For example, the significant rise in carbapenem resistance had become a global issue., 2,6-8 OXA-type carbapenemase-linked enzymatic destruction is the most effective resistance mechanism against carbapenems in $A$. baumannii. ${ }^{9,10}$ While the OXA-51 gene region is intrinsic to $A$. baumannii isolates, the presence of other OXA-type resistance genes such as OXA-23, OXA-24, and OXA-58 changes regionally. 6,9

The multidisciplinary work of the Hospital Infection Control Committee (HICC) is necessary to evaluate epidemics of HAI detected in ICUs. ${ }^{2,3,11}$ Some of the precautions that can be applied are hand hygiene, culture surveillance sampling, a rigorous contact isolation protocol, strict precautions for carriers, patient isolation, cohorting of medical equipments, healthcare workers serving the affected patient group, environmental cleaning, and closing the unit after the outbreak. ${ }^{1-3,11-13}$ For the effective application of these measures, epidemiological typing becomes important in the determination of the source of the epidemic, transmission pathways, and the epidemiological relationship with patients. ${ }^{14,15}$ Although there are several phenotypic and genotypic typing methods available for

Disclosure. Authors have no conflict of interests, and the work was not supported or funded by any drug company. this purpose, PFGE is currently accepted as the "gold standard" among genotyping methods owing to its high discriminatory power and repeatability. ${ }^{15,16}$

The aim of our study is to reveal the relationship between clinical and environmental isolates of A. baumannii, both phenotypically and genotypically, in an $A$. baumannii epidemic in the Anesthesiology and Reanimation ICU and the use of the epidemiological data to determine the epidemic's source, identifying potential risk factors, and to inform the effort to prevent and manage future epidemics.

Methods. Acinetobacter baumannii was isolated from 5 clinical samples (blood, respiratory catheter, bronchoalveolar lavage and 2 respiratory secretion samples) of 3 different patients sent to the medical microbiology laboratory of Sultan Abdulhamid Han Training and Research hospital, Istanbul, Turkey, from the Anesthesiology and Reanimation ICU for a week period between 03 January 2015 and 10 January 2015. During this period 48 clinical samples were sent from 10 patients which were treated in the same ICU. In association with the hospital's HICC, similar cases identified in the Anesthesiology and Reanimation ICU were recognized as a potential outbreak. Established protocols to determine the source and identify potential risk factors were initiated. A total of 59 samples for cultures surveillance were collected from Anesthesiology and Reanimation ICU patients, and from various locations in the environment such as bedsides, patient shelves, monitors, infusion pumps, patient files, refrigerators, dressing carts, phones, defibrillators, the nursing desk, computer keyboards, and the hands of the Anesthesiology and Reanimation ICU staff. After terminal disinfection, control surveillance samples were collected. Infected patients were isolated, and strict contact isolation procedures were initiated. Infected patients, related medical staff, and associated medical devices were cohorted. Hand hygiene and hospital infection education were presented to all hospital staff by the HICC.

Clinical and environmental samples were inoculated onto 5\% sheep's blood agar and eosin methylene blue agar and incubated between $18-24$ hours at $37^{\circ} \mathrm{C}$ in the presence of $5 \% \mathrm{CO}_{2}$. Microbiological identification of $A$. baumannii was achieved using conventional diagnostic tests (oxidase, catalase, sugar fermentation, motility, citrate utilization, and ability to grow at $41^{\circ} \mathrm{C}$ and $44^{\circ} \mathrm{C} .{ }^{17}$ The VITEK ${ }^{\circledast} 2$ automated identification system using the VITEK ${ }^{\oplus} 2$ GN ID card (bioMerieux, France). For further confirmation, PCR was used to detect the intrinsic OXA-51 resistance gene. ${ }^{6,7,9}$ 
The VITEK $^{\oplus} 2$ automated identification system AST-N262 card (Biomerieux, France) was used to determine the antibiotic susceptibility patterns for amikacin, cefepime ceftazidime, ceftriaxone, cefotaxime, ciprofloxacin, colistin, doxycycline, gentamicin, imipenem, levofloxacin, meropenem, minocycline, piperacillin-tazobactam and tobramycin. Results were interpreted according to an identification criteria set for $A$. baumannii by the Clinical and Laboratory Standards Institute (CLSI). ${ }^{18}$ For the extraction of DNA frames using PCR applications, The MagCore ${ }^{\circledR} \mathrm{HF} 16$ automated DNA extracting system and genomic DNA Bacterial Kit (RBC Bioscience, Taiwan) were used according to manufacturer's instructions. For OXA-type carbapenemase screening, primers targeting the OXA-23, OXA-24, OXA-51, and OXA-58 gene regions were used in multiplex PCRs. ${ }^{19,20}$ The primers and estimated PCR DNA segment sizes are shown in (Table 1).

The PFGE analysis of $A$. baumannii isolates was performed after digestion with ApaI enzyme. ${ }^{21,22}$ The relationship between isolates was evaluated by studying band profiles according to criteria developed by Tenover et al. ${ }^{23}$ Open source PyElph software (version 1.4) was used for the analysis. Band tolerance was set at $2 \%$ for the calculation of similarity coefficients. Pulsedfield gel electrophoresis profile dendrograms were constructed, and a cluster analysis was completed with the "unweighted pair-group method with arithmetic averages" approach.

Results. Among the environmental samples, bacterial growth was observed in 3: the patient shelf, patient monitor, and the infusion pump. All 8 isolates obtained from the 5 clinical and 3 environmental sources were identified as $A$. baumannii complex by conventional methods and the VITEK 2 system. All isolates were susceptible to colistin and tobramycin and resistant to cefepime, cefotaxime, ceftazidime, ceftriaxone,

Table 1 - Primers used for PCR applications and expected band sizes.

\begin{tabular}{ccc}
\hline Primers & Sequence (5'-3') & Expected band sizes \\
\hline OXA-23- & GAT CGG ATT GGA GAA CCA GA & 501bp \\
OXA-23- & ATT TCT GAC CGC ATT TCC AT & \\
OXA-24- & GGT TAG TTG GCC CCC TTA AA & 246bp \\
OXA-24- & AGT TGA GCG AAA AGG GGA TT & \\
OXA-51- & TAA TGC TTT GAT CGG CCT TG & 353bp \\
OXA-51- & TGG ATT GCA CTT CAT CTT GG & \\
OXA-58- & AAG TAT TGG GGC TTG TGC TG & 599bp \\
OXA-58- & CCC CTC TGC GCT CTA CAT AC & \\
\hline PCR - polymerase chain reaction, OXA - OXA-type carbapenemases, \\
bp - band size
\end{tabular}

ciprofloxacin, doxycycline, imipenem, levofloxacin, meropenem, minocycline and piperacillin-tazobactam. The clinical and environmental samples of patients $X$ and $\mathrm{Y}$ had phenotypically similar antibiotic susceptibility patterns (Table 2).

All isolates were positive for the OXA-51 and OXA-23 gene regions. None of the isolates were positive for the OXA-24 and OXA-58 gene regions (Figure 1, Table 2).

According to the Tenover criteria, ${ }^{23}$ used for band analysis in molecular typing, 6 of the isolates appeared to have the same band profile and were considered epidemic isolates. Two isolates contained 3 bands that differed from those of the epidemic isolates, and they were most likely epidemic associated isolates or closely related isolates. For the analysis of band images, we used open source coded PyElph software (version 1.4). The cutoff value for determining the PFGE genotype was $85.2 \%$, and we determined that the 8 isolates included in the study formed 2 clusters (Figure 2, Table 2). The clinical and environmental isolates obtained from patients $\mathrm{X}$ and $\mathrm{Y}$ made up the first cluster (6 isolates), the 2 isolates associated with patient $\mathrm{Z}$ formed the second cluster (Figure 2, Table 2).

Discussion. Acinetobacter baumannii is a common pathogen found in ICUs where invasive procedures are frequently performed, broad-spectrum antibiotics are widely used, and immune suppressed patients are regularly treated. ${ }^{9,14,24}$ Acinetobacter baumannii typically colonizes respiratory equipment. It can cause a wide range of HAI including respiratory system and circulatory system infections and is responsible for epidemics of those distinct infections as well. ${ }^{8,14,24}$ In a study by Mezzetesta et $\mathrm{al}^{25} 70.1 \%$ of hospital infections caused by $A$. baumannii were respiratory system infections, and $16.9 \%$ were blood infections. Although this is a descriptive study with few isolates and results obtained from this isolates were not predictive, we roughly found similar percentages (4 respiratory infections- $80 \%$ and one circulatory system infection$20 \%$ ) during the $A$. baumannii epidemic.

Acinetobacter baumannii complex (ABC) includes several bacterial species which are; Acinetobacter calcoaceticus (A. calcoaceticus), A. baumannii, Acinetobacter pittii (A. pittii) 3 species and Acinetobacter nosocomialis (A. nosocomialis) species 13TU.,17 Although these species are included in $\mathrm{ABC}$, they have totally different clinical symptoms, dissemination patterns, antibiotic resistance mechanisms and epidemiology. ${ }^{7}$ For this reason it's very important to discriminate $A$. baumannii from other $\mathrm{ABC}$ species. 
Table 2 - Results of demographic data, antibiotic resistance profiles, OXA genes and PFGE analysis of isolates.

\begin{tabular}{lccccccc}
\hline Lane & Patient & Gender & Age & Site of isolation & $\begin{array}{c}\text { Antibiotic resistance } \\
\text { profiles }\end{array}$ & OXA genes & PFGE analysis \\
\hline 1 & X & Male & 80 & Blood & 1 & OXA-23+OXA-51 & A \\
2 & X & Male & 80 & Respiratory secretion & 1 & OXA-23+OXA-51 & A \\
3 & Y & Female & 78 & Respiratory catheter & 1 & OXA-23+OXA-51 & A \\
4 & Y & Female & 78 & Bronchoalveolar lavage & 1 & OXA-23+OXA-51 & A \\
5 & Y & Female & 78 & Patient shelf & 1 & OXA-23+OXA-51 & A \\
6 & Y & Female & 78 & Infusion pump & 1 & OXA-23+OXA-51 & A \\
7 & Z & Male & 56 & Respiratory secretion & 2 & OXA-23+OXA-51 & B \\
8 & Z & Male & 56 & Patient monitor & 2 & OXA-23+OXA-51 & B \\
\hline \multicolumn{7}{c}{ OXA - OXA-type carbapenemases, PFGE - pulsed-field gel electrophoresis. } \\
\hline \multicolumn{7}{c}{}
\end{tabular}

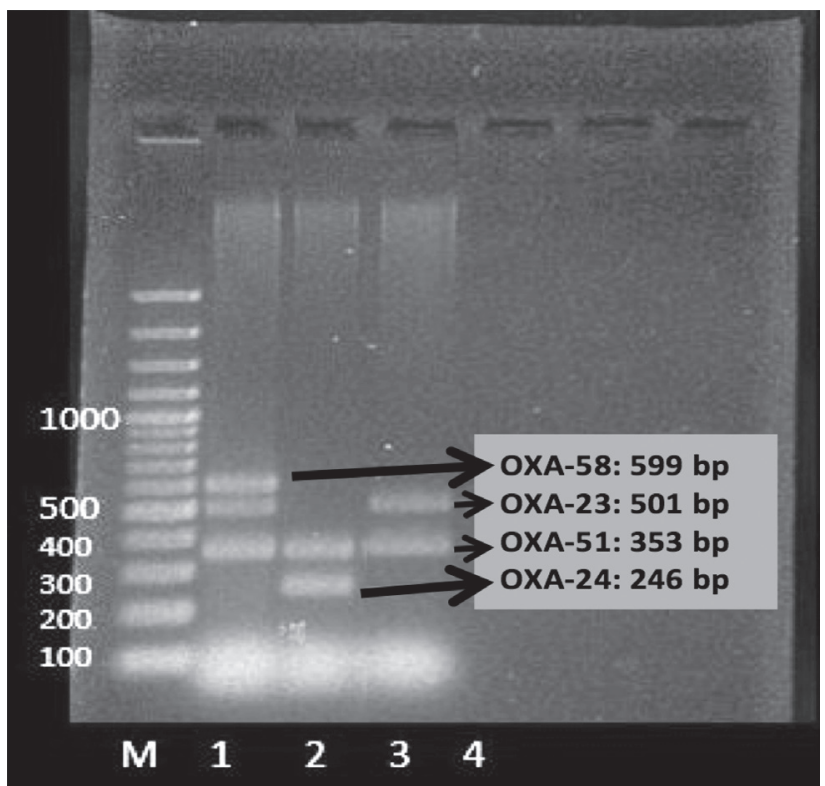

Figure 1 - The detection of OXA-carbapenamase encoding genes by multiplex PCR. M: 100bp DNA Marker. Lane 1: positive control isolate containing OXA-23, OXA-51 and OXA-58 genes, lane 2: positive control isolate containing OXA-24, OXA-51 genes, lane 3: representative image of 8 isolates both containing OXA-23 and OXA-51 genes, lane 4: negative control.

Phenotypic methods and molecular tests are the most widely used systems for identification of $A$. baumannii, but differentiating Acinetobacter species by biochemical methods is difficult. Commercial systems using biochemical properties such as VITEK $^{\oplus}$ cannot discriminate between $\mathrm{ABC}$ species. ${ }^{26}$ In our study, all isolates were identified as $\mathrm{ABC}$ by VITEK ${ }^{\oplus} 2$. Ability to grow at $41^{\circ} \mathrm{C}$ and $44^{\circ} \mathrm{C}$ and intrinsic presence of the OXA-51 gene were used for species differentiation; all isolates were identified as A. baumannii. Several studies indicate that the presence of the OXA-51 resistance gene can be used for identification of $A$. baumannii. ${ }^{6,7,25}$

Development of resistance to carbapenems, which are broad-spectrum beta-lactam antibiotics, is a serious problem when treating Acinetobacter infections. ${ }^{2,9}$ Carbapenem resistance was found in $100 \%$ of A. baumannii isolates in studies performed by Teo et al, ${ }^{27}$ and Hammerun et al. ${ }^{28}$ Similarly, in our study, we found all $A$. baumannii isolates were resistant to imipenem and meropenem minimum inhibitory concentration (MIC) values of $\geq 16 \mathrm{mg} / \mathrm{L}$ from VITEK $^{\oplus} 2$ for both imipenem and meropenem. We identified tobramycin MIC value of $\leq 4 \mathrm{mg} / \mathrm{L}$ from VITEK ${ }^{\oplus}$, and colistin MIC value of $\leq 0.5 \mathrm{mg} / \mathrm{L}$ from $\mathrm{VITEK}^{\oplus} 2$, as the most effective antimicrobial agents, both exhibiting 100\% sensitivity. Previous studies also reported colistin sensitivity of A. baumannii as $100 \%$. $^{25,27,29}$

The expression of OXA-type carbapenemases is the most common resistance mechanism seen in A. baumannii. ${ }^{9} 10$ The multiplex PCR method, which we used to screen for resistance genes, provides the opportunity to search for multiple gene regions with a single reaction. ${ }^{9,15}$ All of the isolates in our study contained OXA-51 and OXA-23 gene regions, as detected by multiplex PCR. It is known that the isolates producing OXA-23 carbapenemase exhibit high levels of imipenem and meropenem hydrolysis. ${ }^{1,6,30}$ In our study, we found all of the isolates positive for both OXA-23 and OXA-51 show carbapenem resistance $(\geq 16 \mathrm{mg} / \mathrm{L})$. In the study of Teo et al, ${ }^{27}$ all 49 isolates which contained both OXA-23 and OXA-51 genes, had carbapenem resistance. Rezaei et $\mathrm{al},{ }^{31}$ reported that the prevalence of OXA-58 containing isolates decreased in $A$. baumannii epidemics in ICUs and the prevalence of OXA-23 increased. This result is most likely due to the higher carbapenemase activity of OXA-23 when compared to that of OXA- $58 .^{25}$ 


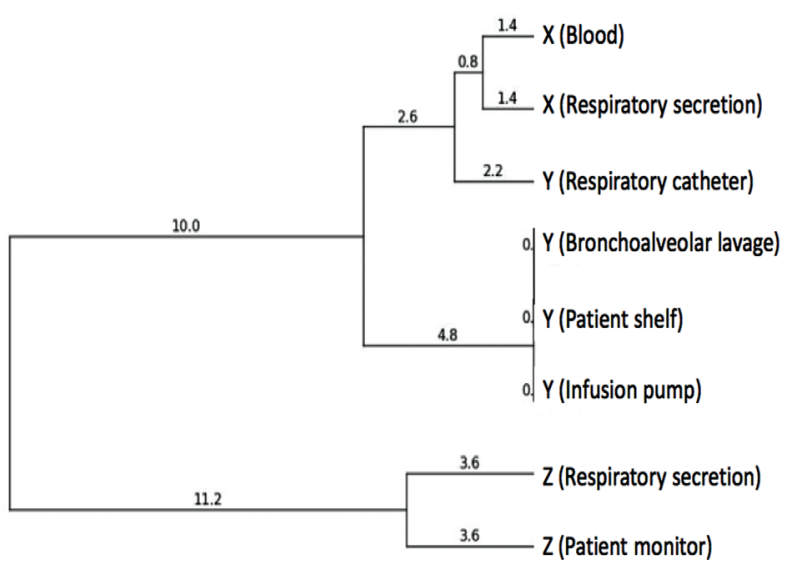

Figure 2 - Results of phylogenetic analysis of isolatescontrol.

The clonal relationships between the bacterial isolates can be demonstrated by molecular typing methods, such as PFGE and multilocus sequence typing (MLST) in epidemiological analyses. ${ }^{1,14-16,28,32}$ Although it has complicated protocols and there are no standard methods for the interpretation and share of data, PFGE is still more widely used 'gold standart' molecular typing method. ${ }^{28,32}$ Multilocus sequence typing which gives significant information for comparison and potential tracing of internationalspreading clones and should replace PFGE typing. ${ }^{28}$ In this study, PFGE analysis was performed to determine clonal relationships, and all isolates were found to be clonally related and could be differentiated into 2 PFGE clusters (A and B) with an 85.2\% similarity coefficient. In other studies, similarity coefficients were set at $80 \%$ for PFGE analysis, which is similar to our study. ${ }^{21,25} \mathrm{Li}$ et al, ${ }^{7}$ reported 33 different PFGE patterns for 145 carbapenem resistant $A$. baumannii isolates and detected 8 different PFGE clones. Keskin et al, ${ }^{19}$ studied a group of 201 Acinetobacter $s p p$. isolates and found they could be categorized into 4 dominant genotypes (A, B, C, and D). Ambrosi et al, ${ }^{33}$ found $31 \mathrm{~A}$. baumannii isolates grouped into 3 subtypes (A, $\mathrm{B}$, and $\mathrm{C})$. Guo-Xin et $\mathrm{al}^{29}$ found $62 \mathrm{~A}$. baumannii isolates representing 9 different clones. Hammerun et $\mathrm{al}^{2}{ }^{28}$ detected 4 different PFGE profiles in 8 investigated isolates and found 4 of the isolates were related to each other. In our study, we found 6 isolates in cluster A (epidemic isolates) and 2 in cluster B (isolates closely related to the epidemic isolates).

Likewise, Çetinkol et $\mathrm{al}^{34}$ showed that all 50 multidrug-resistant $A$. baumannii isolates, except one, were grouped in the same cluster and thought to be epidemic isolates. In a study managed by Karagöz et al, ${ }^{35}$ a total of $54 \mathrm{~A}$. baumannii isolates were collected during an outbreak and when analyzed appeared to originate from a single clone considered responsible for the outbreak. Based on PFGE profiles, we detected that all epidemic isolates had the same OXA-type carbapenemase genes and the phenotypic similarity determined by analyzing antimicrobial susceptibility patterns correlated with genotypic similarity (Table 2).

Because of early intervention of the HICC, the epidemic was successfully controlled without mortalities or closure of the unit. At that time our national and local guidelines were used for infection control procedures with success, but for today we recommend to update these guidelines by the help of a more comprehensive and specific World Health Organization (WHO) guideline for carbapenem-resistant Enterobacteriaceae, A. baumannii and Pseudomonas aeruginosa.

In conclusion, our results suggest that the determination of antimicrobial resistance patterns with daily cultures surveillance follow-ups, identifying resistance gene patterns with amplification-based methods, and the detection of clonal relationships through PFGE analysis are important for the early detection of outbreaks and the rapid implementation of proper countermeasures to stop the spread of the infective agent.

\section{References}

1. Cheng VCC, Wong SC, Chen JHK, So SYC, Wong SCY, Ho PL, et al. Control of multidrug-resistant Acinetobacter baumannii in Hong Kong: Role of environmental surveillance in communal areas after a hospital outbreak. Am J Infect Control 2018; 46: 60-66.

2. WHO Guidelines Approved by the Guidelines Review Committee. Guidelines for the Prevention and Control of Carbapenem-Resistant Enterobacteriaceae, Acinetobacter baumannii and Pseudomonas aeruginosa in Health Care Facilities. Geneva: World Health Organization; 2017.

3. Tanguy M, Kouatchet A, Tanguy B, Pichard É, Fanello S, Joly-Guillou ML. Management of an Acinetobacter baumannii outbreak in an intensive care unit. Med Mal Infect 2017; 47: 409-414.

4. World Health Organization. WHO guidelines on hand hygiene in health care. Geneva $(\mathrm{CN})$ : World Health Organization; 2009.

5. Liu WL, Liang HW, Lee MF, Lin HL, Lin YH, Chen CC, et al. The impact of inadequate terminal disinfection on an outbreak of imipenem-resistant Acinetobacter baumannii in an intensive care unit. PLoS One 2014; 9: e107975.

6. Park YS, Lee H, Lee KS, Hwang SS, Cho YK, Kim HY, et al. Extensively drug-resistant Acinetobacter baumannii: risk factors for acquisition and prevalent OXA-type carbapenemases--a multicentre study. Int J Antimicrob Agents 2010; 36: 430-435. 
7. Li P, Niu W, Li H, Lei H, Liu W, Zhao X, et al. Rapid detection of Acinetobacter baumannii and molecular epidemiology of carbapenem-resistant A. baumannii in two comprehensive hospitals of Beijing, China. Front Microbiol 2015; 6: 997.

8. Cai B, Echols R, Magee G, Arjona Ferreira JC, Morgan G, Ariyasu M, et al. Prevalence of Carbapenem-Resistant GramNegative Infections in the United States Predominated by Acinetobacter baumannii and Pseudomonas aeruginosa. Open Forum Infect Dis 2017; 4: ofx176.

9. Pogue JM, Mann T, Barber KE, Kaye KS. Carbapenemresistant Acinetobacter baumannii: epidemiology, surveillance and management. Expert Rev Anti Infect Ther 2013; 11: 383-393.

10. Ambler RP. The structure of beta-lactamases. Philos Trans $R$ Soc Lond B Biol Sci 1980; 289: 321-331.

11. WHO Guidelines Approved by the Guidelines Review Committee. Guidelines on Core Components of Infection Prevention and Control Programmes at the National and Acute Health Care Facility Level. Geneva: World Health Organization; 2016.

12. Cantey JB, Sreeramoju P, Jaleel M, Treviño S, Gander R, Hynan LS, et al. Prompt control of an outbreak caused by extended-spectrum $\beta$-lactamase-producing Klebsiella pneumoniae in a neonatal intensive care unit. $J$ Pediatr 2013; 163: 672-679. e1-3.

13. Nakamura K, Kaneko M, Abe Y, Yamamoto N, Mori H, Yoshida A, et al. Outbreak of extended-spectrum $\beta$-lactamase-producing Escherichia coli transmitted through breast milk sharing in a neonatal intensive care unit. J Hosp Infect 2016; 92: 42-46.

14. Rafei R, Kempf M, Eveillard M, Dabboussi F, Hamze M, Joly-Guillou ML. Current molecular methods in epidemiological typing of Acinetobacter baumannii. Future Microbiol 2014; 9: 1179-1194.

15. Meshkat Z, Salimizand H, Amini Y, Khakshoor M, Mansouri D, Farsiani $\mathrm{H}$, et al. Molecular characterization and genetic relatedness of clinically Acinetobacter baumanii isolates conferring increased resistance to the first and second generations of tetracyclines in Iran. Ann Clin Microbiol Antimicrob 2017; 16: 51.

16. Goering RV. Pulsed field gel electrophoresis: a review of application and interpretation in the molecular epidemiology of infectious disease. Infect Genet Evol 2010; 10: 866-875.

17. Brisou J, Prevot AR. Family II: Moraxellaceae, Genus II: Acinetobacter. In: Garrity G, Brenner DJ, Krieg NR, Staley JR, editors. Bergey's Manual of Systematic Bacteriology Volume 2: The proteobacteria, Part B: The Gammaproteobacteria. 2th ed. New York (USA): Springer-Verlag; 2005. p. 425-435.

18. Clinical and Laboratory Standards Institute. Performance standards for antimicrobial susceptibility testing. TwentyFourth Informational Supplement, M100-S24. Wayne (PA): Clinical and Laboratory Standards Institute; 2014.

19. Keskin H, Tekeli A, Dolapci İ, Öcal D. Molecular characterization of beta-lactamase-associated resistance in Acinetobacter baumannii strains isolated from clinical samples. Mikrobiyol Bul 2014; 48: 365-376.

20. Woodford N, Ellington MJ, Coelho JM, Turton JF, Ward ME, Brown S, et al. Multiplex PCR for genes encoding prevalent OXA carbapenemases in Acinetobacter spp. Int J Antimicrob Agents 2006; 27: 351-353.

21. Durmaz R, Otlu B, Koksal F, Hosoglu S, Ozturk R, Ersoy Y, et al. The optimization of a rapid pulsed-field gel electrophoresis protocol for the typing of Acinetobacter baumannii, Escherichia coli and Klebsiella spp. Jpn J Infect Dis 2009; 62: 372-377.
22. Turabelidze D, Kotetishvili M, Kreger A, Morris JG Jr, Sulakvelidze A. Improved pulsed-field gel electrophoresis for typing vancomycin-resistant enterococci. J Clin Microbiol 2000; 38: 4242-4245.

23. Tenover FC, Arbeit RD, Goering RV, Mickelsen PA, Murray BE, Persing DH, et al. Interpreting chromosomal DNA restriction patterns produced by pulsed-field gel electrophoresis: criteria for bacterial strain typing. J Clin Microbiol 1995; 33: 2233-2239.

24. Jamal S, Atrouni AA, Rafei R, Dabboussi F, Hamze M, Osman M. Molecular mechanisms of antibiotic resistance in Acinetobacter baumannii with a special focus on its epidemiology in Lebanon. J Glob Antimicrob Resist 2018; 18.

25. Mezzatesta ML, Caio C, Gona F, Cormaci R, Salerno I, Zingali $\mathrm{T}$, et al. Carbapenem and multidrug resistance in Gramnegative bacteria in a single centre in Italy: considerations on in vitro assay of active drugs. Int J Antimicrob Agents 2014; 44: 112-116.

26. Nemec A, Krizova L, Maixnerova M, van der Reijden TJ, Deschaght P, Passet V, et al. Genotypic and phenotypic characterization of the Acinetobacter calcoaceticusAcinetobacter baumannii complex with the proposal of Acinetobacter pittii sp. nov. (formerly Acinetobacter genomic species 3) and Acinetobacter nosocomialis sp. nov. (formerly Acinetobacter genomic species 13TU). Res Microbiol 2011; 162: 393-404.

27. Teo J, Lim TP, Hsu LY, Tan TY, Sasikala S, Hon PY, et al. Extensively drug-resistant Acinetobacter baumannii in a Thai hospital: a molecular epidemiologic analysis and identification of bactericidal Polymyxin B-based combinations. Antimicrob Resist Infect Control 2015; 4: 2.

28. Hammerum AM, Hansen F, Skov MN, Stegger M, Andersen PS, Holm A, et al. Investigation of a possible outbreak of carbapenem-resistant Acinetobacter baumannii in Odense, Denmark using PFGE, MLST and whole-genome-based SNPs. J Antimicrob Chemother 2015; 70: 1965-1968.

29. Guo-Xin M, Dan-Yang S, Xi-Zhou G, Jun-Chang C, Rui W, Zhi-Gang C, et al. Laboratory to Clinical Investigation of Carbapenem Resistant Acinetobacter baumannii Outbreak in a General Hospital. Jundishapur J Microbiol 2014; 7: e13120.

30. Abdallah M, Olafisoye O, Cortes C, Urban C, Charles C, Landman D, et al. Reduction in the prevalence of carbapenemresistant Acinetobacter baumannii and Pseudomonas aeruginosa in New York City. Am J Infect Control 2015; 43: 650-652.

31. Rezaei A, Fazeli H, Moghadampour M, Halaji M, Faghri J. Determination of antibiotic resistance pattern and prevalence of OXA-type carbapenemases among Acinetobacter baumannii clinical isolates from inpatients in Isfahan, central Iran. Infez Med 2018; 26: 61-66.

32. Noormohamed A, Fakhr MK. Molecular Typing of Campylobacter jejuni and Campylobacter coli Isolated from Various Retail Meats by MLST and PFGE. Foods 2014; 3: 82-93.

33. Ambrosi C, Aleandri M, Giordano A, Scribano D, Marazzato M, Zagaglia C, et al. Molecular characterisation of extensively drug-resistant Acinetobacter baumannii: First report of a new sequence type in Italy. J Glob Antimicrob Resist 2016; 7: 154-156.

34. Çetinkol Y, Telli M, Altunçekiç Yıldırım A, Çalgın MK. Evaluation of the efficacy of colistin/sulbactam combination on carbapenem-resistant Acinetobacter baumannii strains. Mikrobiyol Bul 2016; 50: 460-465.

35. Karagöz A, Baran I, Aksu N, Acar S, Durmaz R. [Characterization and determination of antibiotic resistance profiles of a single clone Acinetobacter baumannii strains isolated from blood cultures]. Mikrobiyol Bul 2014; 48: 566-576. Turkish 